\title{
Semiconductor Laser Diode Pumps for Inertial Fusion Energy Lasers
}

R. Deri, J. Geske, M. Kanskar, S. Patterson, G. Kim, Q. Hartmann, F. Leibreich, E. Deichsel, J. Ungar,P. Thiagarajan, R. Martinsen, $P$. Leisher, E. Stephens, J. Harrison, C. Ghosh, O. Rabot, A. Kohl

January 2011 


\section{Disclaimer}

This document was prepared as an account of work sponsored by an agency of the United States government. Neither the United States government nor Lawrence Livermore National Security, LLC, nor any of their employees makes any warranty, expressed or implied, or assumes any legal liability or responsibility for the accuracy, completeness, or usefulness of any information, apparatus, product, or process disclosed, or represents that its use would not infringe privately owned rights. Reference herein to any specific commercial product, process, or service by trade name, trademark, manufacturer, or otherwise does not necessarily constitute or imply its endorsement, recommendation, or favoring by the United States government or Lawrence Livermore National Security, LLC. The views and opinions of authors expressed herein do not necessarily state or reflect those of the United States government or Lawrence Livermore National Security, LLC, and shall not be used for advertising or product endorsement purposes.

\section{Auspices Statement}

This work performed under the auspices of the U.S. Department of Energy by Lawrence Livermore National Laboratory under Contract DE-AC52-07NA27344. 


\title{
Semiconductor Laser Diode Pumps for Inertial Fusion Energy Lasers
}

\author{
Dr. Robert Deri, Chief Technologist Engineering, Lawrence Livermore National Laboratory, Livermore CA \\ Dr. Jon Geske, President, Aerius Photonics LLC, Ventura CA \\ Dr. Manoj Kanskar, Vice President of Research and Development, Alfalight Inc., Madison WI \\ Dr. Steve Patterson, Technical Director, DILAS Laser Diode Inc., Tucson AZ \\ Dr. Gerald Kim, President, EOTRON LLC, Oceanside CA \\ Dr. Quesnell Hartmann, CEO, Epiworks, Inc., Champaign, IL \\ Dr. Franck Leibreich, Director of Sales and Marketing, Semiconductor Products, IPG Photonics, Santa Clara CA \\ Dr. Eckard Deichsel, R\&D Team Manager Diode Lasers, JENOPTIK Laser GmbH, Jena, Germany \\ Dr. Jeffrey Ungar, Chief Technology Officer, Laser Operations LLC/QPC Lasers Division, Sylmar CA \\ Mr. Prabhu Thiagarajan, Vice President of Engineering, Lasertel Inc., Tucson AZ \\ Dr. Rob Martinsen, Vice President of Product Engineering, nLight Corporation, Vancouver VA \\ Dr. Paul Leisher, Manager of Advanced Technology, nLight Corporation, Vancouver VA \\ Dr. Edward F. Stephens, General Manager, Northrup Grumman - Cutting Edge Optronics, St. Charles MO \\ Dr. Jim Harrison, Sr. Director of Semiconductor Laser Technology, Oclaro, Oro Valley AZ \\ Dr. Chuni Ghosh, Chief Technology Officer, Princeton Optronics, Mercerville NJ \\ Mr. Olivier Rabot, Laser Diodes Director, Quantel, Les Ulis, France \\ Dr. Andreas Kohl, R\&D Manager, Quantel, Les Ulis, France
}

\section{Introduction and Overview}

Solid-state lasers have been demonstrated as attractive drivers for inertial confinement fusion on the National Ignition Facility (NIF) at Lawrence Livermore National Laboratory (LLNL) and at the Omega Facility at the Laboratory for Laser Energetics (LLE) in Rochester, NY. For power plant applications, these lasers must be pumped by semiconductor diode lasers to achieve the required laser system efficiency, repetition rate, and lifetime. Inertial fusion energy (IFE) power plants will require approximately 40 -to- $80 \mathrm{GW}$ of peak pump power, and must operate efficiently and with high system availability for decades. These considerations lead to requirements on the efficiency, price, and production capacity of the semiconductor pump sources. This document provides a brief summary of these requirements, and how they can be met by a natural evolution of the current semiconductor laser industry.

The detailed technical requirements described in this document flow down from a laser amplifier design described elsewhere. [1] In brief, laser amplifiers comprising multiple Nd:glass gain slabs are facepumped by two planar diode arrays, each delivering 30 to $40 \mathrm{MW}$ of peak power at $872 \mathrm{~nm}$ during a 200 $\mu$ s quasi-CW (QCW) pulse with a repetition rate in the range of 10 to $20 \mathrm{~Hz}$. The baseline design of the diode array employs a 2D mosaic of submodules to facilitate manufacturing. As a baseline, we envision that each submodule is an array of vertically stacked, $1 \mathrm{~cm}$ wide, edge-emitting diode bars, [e.g.; 2] an industry standard form factor. These stacks are mounted on a common backplane providing cooling and current drive. Stacks are conductively cooled to the backplane, to minimize both diode package cost and the number of fluid interconnects for improved reliability. While the baseline assessment in this document is based on edge-emitting devices, the amplifier design does not preclude future use of surface emitting diodes, $[3,4]$ which may offer appreciable future cost reductions and increased reliability.

The high-level requirements on the semiconductor lasers involve reliability, price points on a price-perWatt basis, and a set of technical requirements. The technical requirements for the amplifier design in Ref. [1] are discussed in detail below and are summarized in Table I. These values are still subject to changes as the overall laser system continues to be optimized. Since pump costs can be a significant 
fraction of the overall laser system cost, it is important to achieve sufficiently low price points for these components. At this time, our price target for tenth-of-a-kind IFE plant is $\$ 0.007 / \mathrm{Watt}$ for packaged devices. At this target level, the pumps account for approximately one third of the laser cost. The pump lasers should last for the life of the power plant, leading to a target component lifetime requirement of roughly 14 Gshots, corresponding to a 30 year plant life and $15 \mathrm{~Hz}$ repetition rate.

An attractive path forward involves pump operation at high output power levels, on a Watts-per-bar (Watts/chip) basis. This reduces the cost of pump power (price-per-Watt), since to first order the unit price does not increase with power/bar. The industry has seen a continual improvement in power output,[5] with current $1 \mathrm{~cm}$-wide bars emitting up to $500 \mathrm{~W}$ QCW (quasi-continuous wave). Increased power/bar also facilitates achieving high irradiance in the array plane. On the other hand, increased power implies greater heat loads and (possibly) higher current drive, which will require increased attention to thermal management and parasitic series resistance. Diode chips containing multiple p-n junctions and quantum wells (also called nanostack structures) may provide an additional approach to reduce the peak current. [6]

TABLE I. Diode Pump Subsystem Requirements and Operating Parameters

\begin{tabular}{|l|c|l|}
\hline Item & Value & \multicolumn{1}{c|}{ Comments } \\
\hline Array Size & $53 \times 27 \mathrm{~cm}^{2}$ & \\
Array Peak Power & $36 \mathrm{MW}$ & \\
Diode wavelength range & $872 \pm 6.6 \mathrm{~nm}$ & Without polarization multiplexing.* $^{*}$ \\
Array Irradiance* & $20 \mathrm{~kW} / \mathrm{cm}^{2}$ & Baseline value, may increase with laser optimization $^{*}$ \\
Pulse width & $175 \mu \mathrm{s}$ & Including fast-axis collimator \\
Diode efficiency & $72 \%$ & Laser optimization may increase to $20 \mathrm{~Hz}$ \\
Repetition Rate & $15 \mathrm{~Hz}$ & \\
Duty cycle & $<0.5 \%$ & \\
Diode fast axis divergence & 4 degrees FW 1/e & \\
Life time & $\sim 14 \mathrm{Gshots}$ & Target value; to 20\% power reduction \\
\hline
\end{tabular}

* The array irradiance is less than the irradiance required of the stack submodules, due to the space between stacks within an array. Required stack irradiance is estimated to be $25 \mathrm{~kW} / \mathrm{cm}^{2}$.

\section{Technical Requirements}

The wavelength requirement for IFE pumps is determined by the absorption spectrum of the gain medium. The $872 \mathrm{~nm} \mathrm{Nd:glass} \mathrm{absorption} \mathrm{peak} \mathrm{is} \mathrm{close} \mathrm{to} \mathrm{the} \mathrm{operating} \mathrm{wavelength} \mathrm{of} \mathrm{commercial}$ semiconductor lasers used for pumping $(880 \sim 885 \mathrm{~nm})$ and medical applications $(870 \mathrm{~nm})$. [7] These devices are produced using InGaAlAs or InGaAsP epitaxial layers on laser grade GaAs substrates, which are available in diameters up to four inches. Their wavelength can be tuned to $872 \mathrm{~nm}$ by small changes in the device epitaxy. [e.g.; 8,9] The required wavelength control $( \pm 6.6 \mathrm{~nm})$ is set by the amplifier configuration: the relatively long absorption path of the Ref. [1] design provides rather loose wavelength control requirements. Since wavelength tolerances of $\pm 3 \mathrm{~nm}$ can be routinely achieved, this requirement is not expected to impact production yields or costs.

High pump irradiance facilitates coupling pump light to the amplifier gain slabs, and enables a more compact beam line design. A diode pitch from 200 to $400 \mu \mathrm{m}$ is required to achieve $20 \mathrm{~kW} / \mathrm{cm}^{2}$ in the array plane for diode powers from 500 to $1000 \mathrm{~W} / \mathrm{bar}$. As mentioned above, $500 \mathrm{~W} / \mathrm{bar}$ QCW devices are already commercially available, and $1000 \mathrm{~W} / \mathrm{bar} \mathrm{CW}$ has been demonstrated in several research publications.[9,10] Stacks with bar pitch $\leq 200 \mu \mathrm{m}$ have been described in the literature $[2,11]$ and are commercially available as products with pitches as low as $300 \mu \mathrm{m}[12]$ using diode-on-submount 
technology and $150 \mu \mathrm{m}$ pitch without submounts [11]. We therefore anticipate that this requirement can be met, although some development of stack packages optimized for IFE applications will likely be required (e.g. optimization of beam shaping optics allowing the required collimation at a low pitch). We anticipate that today's standard QCW package, which are typically based on $\mathrm{CuW}$ or $\mathrm{BeO}$ heat spreading submounts, may be replaced with alternative substrates that facilitate microlens alignment and attachment, and/or enable improved thermal management.

Pump efficiency requirements are primarily driven by the goal of optimizing overall laser efficiency. Our target efficiency for IFE application is $72 \%$, which requires some improvement over current commercial devices in the 870 890 nm region. Current state-of-the-art devices have primarily been optimized for $\mathrm{CW}$ applications in the 808 and 940 980 nm wavelength bands, with research demonstrations of $>70 \%$ wallplug efficiency at $808 \mathrm{~nm}$ and 940 980 nm.[13-15] These devices were optimized for CW, lowerpower operation, rather than for high power, QCW operation. Under QCW conditions, efficiencies of $65 \%$ at $808 \mathrm{~nm}$ and at $300 \mathrm{~W}$ peak power have already been demonstrated [16]. Some further development will be required to optimize device designs for higher efficiency operation in IFE applications. Back-ofthe-envelope calculations suggest that our target efficiency can be achieved with epitaxial structures optimized for low series resistance, cavity absorption $<0.7 \mathrm{~cm}^{-1}$ (an experimentally demonstrated value [9]), and cavity lengths of 2 4 mm.[1] More sophisticated simulations, which include $\mathrm{k} \cdot \mathrm{p}$ calculations of gain and radiative recombination, carrier drift-diffusion transport, and quantum well capture/escape dynamics, also suggest that our efficiency targets are achievable at high output power. Simulation results are shown in Fig. 1.
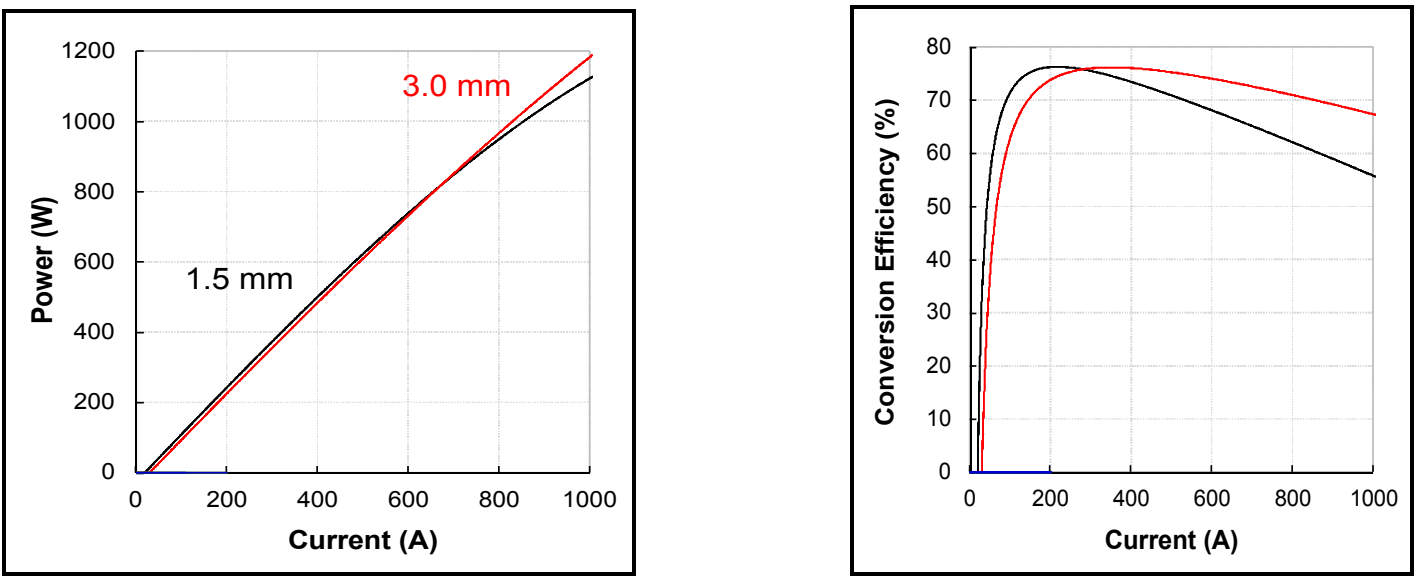

Fig. 1: Simulated power-current and efficiency curves for an IFE-optimized semiconductor laser design. This particular design employs $80 \%$ fill factor and a $3 \mathrm{~mm}$ cavity length (red curve) to achieve efficiencies $>70 \%$ at output powers above $500 \mathrm{~W} / \mathrm{bar}$. While simulations match experimental results obtained for other devices using the same epilayer design used in these simulations, the particular device geometry in the figure has not yet been tested experimentally. (Courtesy P. Leisher, nLight Corp.).

\section{Device Lifetime/Failure Rate}

Edge-emitting semiconductor lasers operating at $\sim 300 \mathrm{~W} /$ bar on $25 \mathrm{C}$ heat sinks have demonstrated lifetimes $>3$ Gshots,[17] and are expected to exhibit 20 Gshot lifetimes.[18] These results suggest that our target device lifetime requirement is achievable. While operation at higher power might negatively impact device lifetime, we anticipate that this will be mitigated by improved device efficiency (lower heat load), short pulse width ( $<200 \mu$ s nominal) compared to usual 200 to $1000 \mu$ s width, and increased cavity 
length (reduced areal heat load in $\mathrm{W} / \mathrm{cm}^{2}$ ). Operation of IFE pumps with significantly colder heat sinks ( $\sim 2 \mathrm{C}$ coolant) is also planned,[1] to achieve improvements in both reliability[19] and efficiency, and will result in average junction temperatures below 27 C.[1] Based on considerations of this type, we anticipate that the IFE lifetime requirement can be achieved with edge-emitting devices. Further improvements in reliability should be achievable with devices that minimize optical intensity at absorptive free semiconductor surfaces (e.g.; edge-emitter facets) where optical damage can occur. Such enhancements can be achieved with edge emitters employing "nonabsorbing mirrors" created by material modifications near the emitting facet, and by surface-emitting devices, which emit over a broader area through nonabsorbing mirrors.

\section{Production Capacity}

The state of the semiconductor laser industry is periodically documented in the trade press and other literature. [e.g.; 5,20,21] Based on reported annual consumption of epitaxial wafers for the entire laser diode market, and assuming a specific chip size and power $\left(2 \times 10 \mathrm{~mm}^{2}, 500 \mathrm{~W} / \mathrm{bar}\right)$, the epitaxy-limit on worldwide capacity for high-power semiconductor lasers is estimated to be roughly $80 \mathrm{GW} / \mathrm{annum}$ in 2009. This estimate assumes that all laser diode production is allocated to high-power devices, which is not the current situation. Since less than $10 \%$ of the diode laser market (by epiwafer areal consumption) involves high power devices, the production capacity relevant for IFE is significantly smaller at this time. Comparison to the $40 \sim 80 \mathrm{GW}$ of peak pump power required for an IFE plant indicates that a substantial increase in production capacity will be required. Capacity increases can also be obtained by leveraging the capabilities of merchant epitaxy suppliers. Other industries that have reached the scale envisioned for IFE laser diodes have utilized these organizations for scaling production and producing epitaxial wafers in high volumes and low costs.

In addition to epitaxial wafer production, capacity will also be limited by the ability to package the semiconductor laser chips. Today's packaging technology and capacity have evolved to meet current market demands, and has yet to fully exploit all technologies available for higher volume production. These include the use of diode heat spreading submounts with mechanical fiducials and structures to facilitate precision alignments (e.g.; technologies analogous to the "silicon optical bench" technology used for low power fiber telecom components) and related technologies for hybrid packaging.[23] They also include the use of high-throughput automation, which can significantly increase packaging throughput for laser diodes, much as it does for silicon VLSI chips.[24,25] Finally, the use of surfaceemitting semiconductor laser devices[3,4] may also enable increased packaging capacity, due to potential simplifications of the required packaging and testing.

To place the capacity increase required for IFE in perspective, it is worth considering recent increases in LED production. Prompted by market demands for display backlights, industry added over 100 MOCVD reactors to increase LED epitaxial growth in one year (some manufacturers placed orders for 100 MOCVD tools in one year). [22] In terms of high-power diode capacity, this quantity of production tools corresponds to $\sim 100 \mathrm{GW}$ of peak diode power production/year. While LED and high-power laser processes are not identical, this example clearly illustrates that capacity can be scaled given the appropriate market drivers. Therefore, we anticipate that capacity scaling to support IFE power production is feasible, given sufficient lead time. Due to the significant scale up required for IFE, several years will likely be required for this facilitization. 


\section{Achievable Price Points}

Any discussion of pump price points must consider the historical improvement in semiconductor laser prices over time, the increases in power/bar over time, and the dependence of pricing on both order volume and production rate. For example, the higher power possible for QCW pulsed operation in IFE applications results in lower pump costs (price/Watt) than is achievable for CW operation. Most significantly, the high volume of semiconductor lasers required for IFE will result in substantial price decreases on a price/Watt basis. As discussed above, one IFE plant will consume at least 100x more semiconductor lasers than are produced annually by any one company at the present time. Since current production infrastructure has been optimized to meet today's market volumes, today's price points are not representative of those achievable at IFE volumes. At higher volumes, we anticipate that semiconductor laser manufacturing will employ methods to both increase capacity and reduce cost that are similar to those used in high-volume silicon VLSI manufacturing. These include transitioning to larger diameter semiconductor wafers (current laser diode fabrication primarily uses 2 to 4 inch diameter wafers, in contrast to the 6 inch wafers used for GaAs electronic devices)[26] and increased use of automation for the handling, mounting, and stacking of individual laser chips, [24,25] the use of improved submount structures to facilitate chip mounting and microlens attachment,[23] as well as increased automation for the testing of final assemblies.

Five of us have independently performed studies to assess achievable semiconductor laser price points. These studies leveraged our internal expertise in the manufacture of edge emitting laser diodes, and employed production models that consider the relevant process steps, include both labor and capital costs. Each evaluation assumed a somewhat different build quantity, but all assumed a single, one-time build. Since each assessment was based on a different power per bar and on somewhat different stacked yields (due to the independent nature of these efforts), each assessment was normalized to a common power per bar $(500 \mathrm{~W})$ and stacked yield (80\%) for comparison.[27] The normalized results range from $\$ 0.018$ to $\$ 0.033 /$ Watt, in rather close agreement given the variety of technology assumptions, production methods, and production rates assumed in the assessments.

Figure 2 summarizes these results. Since the assessments assumed a single build of one IFE plant, with diode production spanning multiple years, we have also extrapolated the results to the case of sustained production of multiple plants using the cost vs. production rate scaling established in [28]. These results are illustrated by the green curves in Fig. 2, for a range of scaling exponents.[29] They indicate that the target of $0.7 \varnothing / \mathrm{W}$ target for $\mathrm{n}^{\text {th }}$-of-a-kind IFE plants is achievable. Since these price points scale with the power per laser chip, additional price reductions should be achievable if the device power can be increased above the $500 \mathrm{~W} / \mathrm{cm}$-bar value used in Fig. 2. This provides a motivation for continued development of higher power devices. 


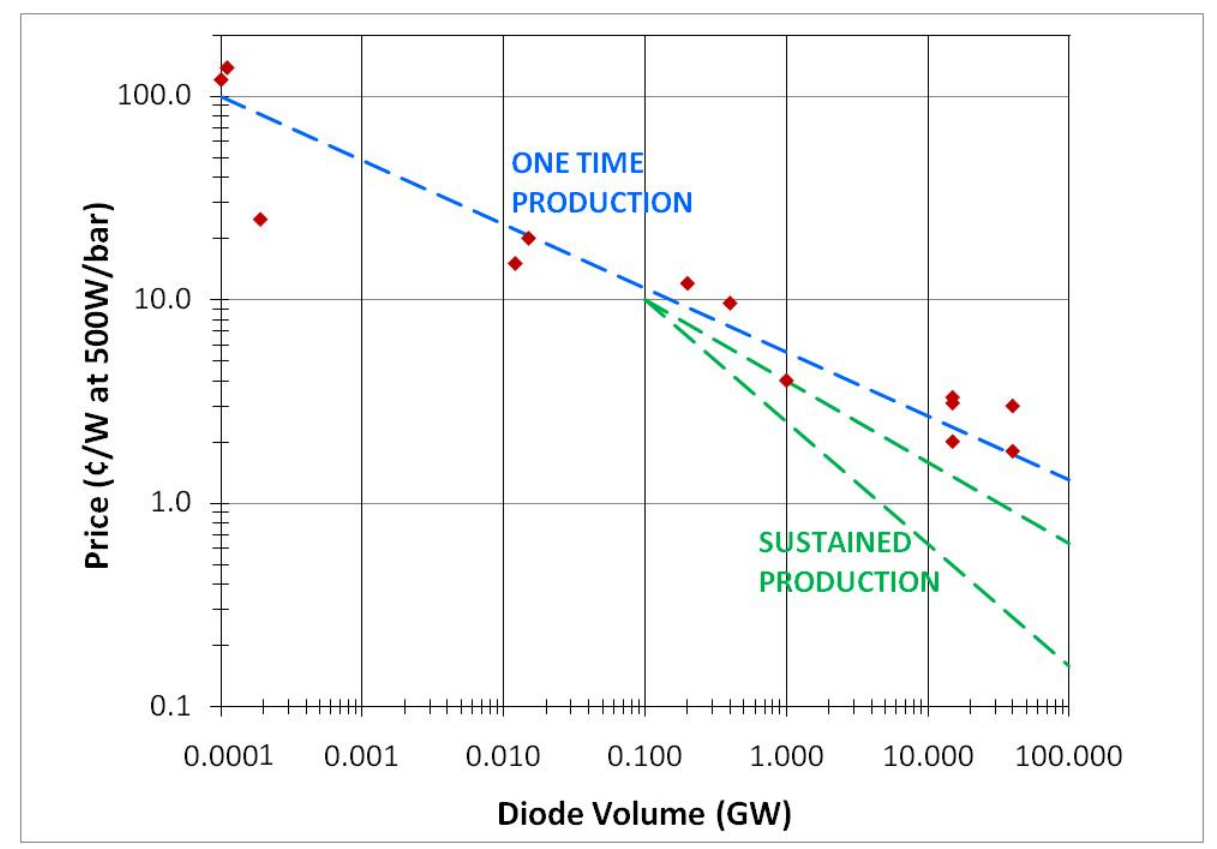

Fig. 2: Summary of high-volume price assessments. Points represent study results for edge emitting diodes, assuming an output power of $500 \mathrm{~W} / \mathrm{bar}$. Prices will scale with the reciprocal of this power. The dashed blue line provides a guide for the eye to these results. The dashed green shows the range expected for sustained production based on the methods of [28], as described in the text.

In addition to edge-emitter assessments, two of us have also evaluated the price points that could be achievable with surface emitter technology [3-4] These studies suggest that these technologies could further reduce the price/Watt of IFE pumps, by a factor of up to 1.4 to 3, providing motivation for continued development of this technology.

\section{Summary}

A consideration of IFE semiconductor laser pump requirements shows that these requirements are achievable, from both a technical and cost perspective. Customizing existing technology for the IFE application will require some engineering, and there will be a lead time associated with this effort. Achieving the production capacity and price points required for IFE will require substantial changes and additions to current laser diode manufacturing infrastructure. While we anticipate that capacity and price targets will be achievable, several years will be required to build the facilities necessary to reach these goals. 


\section{References}

1. A. BAYRAMIAN, et al., $19^{\text {th }}$ Topical Mtg. Fusion Energy (ANS, Las Vegas,NV; November 2010), Paper T3-OS-12-1; to appear in Fusion Sci. Technol. (2011)

2. D. SCHLEUNING et al., Proc. SPIE 7198, 719802 (2009)

3. M. KANSKAR et al, Tech. Digest $22^{\text {nd }}$ Sol. St. Diode Laser Technol. Review 41 (2005)

4. J.-F. SEURIN et al., Proc. SPIE 6876, 68760D-1 (2008)

5. T. HAUSKEN, Laser Focus World 42, 69 (August 2006).

6. M. MULLER et al., Proc. SPIE 6456, 64561B-1 (2007)

7. A.S. LANDSMAN, J. Am. Podiatric Med. Assoc. 100, 166 (2010)

8. P. LEISHER et al., Proc. SPIE 7325, 73250R-1 (2009)

9. H. LI et al., Proc. SPIE 6876, 68760G-1 (2008); Photon. Technol. Lett. 19, 960 (2007)

10. D. SCHROEDER et al., Proc. SPIE 6456, 64560N-1 (2007)

11. R.FEELER and E. STEPHENS, Proc. SPIE 7686, 7860M-1 (2010)

12. QUANTEL, standard QCW product with $300 \mu \mathrm{m}$ pitch @ $100 \mathrm{~mJ}$ pulse

13. P. CRUMP et al., Photon. Technol. Lett. 20, 1378 (2008)

14. P. CRUMP et al., Proc. SPIE 6397, 639706 (2006)

15. M. KANSKAR et al, Electron. Lett. 41, 245 (2005)

16. A. KOHL et al., Proc. SPIE 7835, 2010

17. E. DEICHSEL et al., Proc. SPIE 6876, 68760K (2008).

18. QUANTEL, Olivier Rabot, private communication based on reliability tests for the ESA's ADMAeolus ALADIN instrument (http://www.esa.int/esaLP/SEMQT7ARR1F LPadmaeolus 0.html).

19. H. KISSEL et al., Proc. SPIE 6876, 687618 (2008)

20. R. SZWEDA, III-Vs Review 15, 42 (2002).

21. R.V. STEELE, Laser Focus World 44(2), 69 (February 2008).

22. M. LAPEDUS, "Next Fab Wave: LEDs", EE Times (April 7, 2010)

23. H.P. ZAPPE, Laser Diode Microsystems (Springer, New York; 2004). Ch. 7.

24. D.D. EVANS and Z. BOK, "Micron Level Placement Accuracy for Optoelectronic Components", $59^{\text {th }}$ Electron. Comp. Technol. Conf. (IEEE, May 2009) and related Palomar Technologies eBook at http://palomartechnologies.com/Applications/LaserDiodePackaging.aspx

25. John WALLACE, "Assembly of high-power laser diodes is automated for the first time", Laser Focus World 45, (October 2009).

26. Current production is primarily based on 2 to 3 " wafers; 4 " laser-quality wafers are available today, and cell phone components are routinely produced on 6" GaAs wafers.

27. The price/Watt scales with power/bar to first order, since the cost per unit does not vary with chip power. The $500 \mathrm{~W} / \mathrm{bar}$ value was selected based on the same considerations described in the discussion of irradiance and technical requirements.

28. C.T. GODDARD, IEEE Trans. Comp. Hybrids, Manufact. Technol. CHMT-5, 328 (1982)

29. Opportunity curve exponents of 0.4 to 0.6 were used in Fig. 2. 